\title{
Programmable Reconfigurable Self-Assembly: Parallel Heterogeneous Integration of Chip-Scale Components on Planar and Nonplanar Surfaces
}

\author{
Jaehoon Chung, Wei Zheng, Member, IEEE, Thomas J. Hatch, and Heiko O. Jacobs, Member, IEEE
}

\begin{abstract}
This paper reports on a programmable reconfigurable self-assembly (PRS) process to enable heterogeneous integration of components on nonplanar substrates. The proposed process makes use of solder-based receptors that can be activated electrically. Metal contacts on segmented semiconductor devices bind to liquid-solder-based-receptors on a substrate during the fluidic self-assembly. Programmability is implemented using solder-based receptors that can be switched "ON" and "OFF" using integrated heaters. We have evaluated the feasibility of the proposed PRS concept through computer simulations using ANSYS to estimate: i) the necessary power to heat selected receptors to above the melting point of the solder and ii) the minimal spacing between receptors for preventing thermal crosstalk. A prototype platform has been fabricated to experimentally test the PRS process. The programmable sequential assembly of multiple types of components onto target positions has been demonstrated, including 300- $\mu \mathrm{m}$-sized light-emitting diodes (LEDs) and silicon dies. Three types of defects were identified and eliminated using improved component designs, transient heating, and adequate heat sinks. A prototype color LED display segment that contains a total of 36 red, green, and yellow LED segments and 72 interconnects has been used to test the concept. The outlined process provides a new concept to the parallel integration of microdevices and systems that require electrical interconnects between components.

[1564]
\end{abstract}

Index Terms-Directed assembly, heterogeneous integration, programmable microelectromechanical systems (MEMS), reconfigurable self-assembly, sequential self-assembly, solder-based receptor, template assembly.

\section{INTRODUCTION}

$\mathbf{F}$ ABRICATION strategies that rely on mechanisms of self-assembly are widely recognized as inevitable tools in nanotechnology. Self-assembly, however, is not limited to the nanometer length scale. Strategies that are based on self-assembly are projected to have a major impact in the manufacturing of systems on both the micrometer and nanometer length scale [1], [2]. Several directed self-assembly methods have been demonstrated to generate functional electrical microsystems. For example, Smith et al. reported shape-directed fluidic methods to position light-emitting diodes (LEDs) onto silicon substrates [3]. The authors employed wet-etching

Manuscript received April 2, 2005; revised September 26, 2005. This work was supported by the National Science Foundation under Grant ECS0300263. Subject Editor E. Obermeier.

The authors are with the Department of Electrical Engineering, University of Minnesota, Minneapolis, MN 55455-0167 USA (e-mail: chun0174@umn.edu; hjacobs@ece.umn.edu).

Digital Object Identifier 10.1109/JMEMS.2006.872226 properties of silicon and gallium arsenide to form trapezoidal holes and components. The trapezoidal dies prevent upside down assembly and settled in the openings due to gravity and shape recognition. The process works well on planar substrates and accomplishes filling factors that exceed 99\%. Potential disadvantages are that electrical interconnects requires further processing and that the trapezoidal dies are not commonly used in the industry. Srinivasan et al. developed a different process to assembled silicon components and micromirrors onto a gold-coated silicon substrate [4]-[6]. The authors used hydrophobic patterns on hydrophilic substrates to direct the assembly process of the components; hydrophobic parts attached to the hydrophobic regions to minimize the interfacial free energy. Alignment and bonding was achieved using an adhesive lubrication layer and the defect free assembly of 98 parts, $500 \mu \mathrm{m}$ in size, was accomplished with $0.3^{\circ}$ rotational precision. Gracias et al. demonstrated the formation of three-dimensional (3-D) electrical networks [7] by adapting a liquid solder directed self-assembly process [8]. The authors demonstrated the defect free assembly of 3-D electrical networks that contained 12 identical polyhedra. The $\sim 1 \mathrm{~cm}$ sized polyhedra carried solder coated sheets of printed circuit board material that was taped onto the faces by hand. Jacobs et al. developed a solder directed self-assembly process to assemble semiconductor dies onto substrates [9]. The process did not use solder on the components that were assembled. Instead, it used metal-coated contacts (binding sides) on the dies and solder-based receptors on a substrate to direct the assembly and to form electrical interconnects. That process used standard die geometries and substrates that were fabricated using parallel micromachining. The authors demonstrate the assembly of 1500 silicon dies, $300 \mu \mathrm{m}$ in size, and a defect free cylindrical display segment that contained an array of 113 LEDs.

All of the mentioned methods share the advantage of being massively parallel when compared to robotic assembly. Surface tension directed methods [4]-[9] work in 3-D and on curved substrates and can potentially be scaled down to much smaller dimensions; process limitations, parameters, and design rules, however, have not yet been established. The surveyed methods, when compared to robotic assembly lines, lack the ability to realize heterogeneous systems that contain many different parts [10]. While the procedures [3]-[6], [9] allow the positioning of a large number of identical components onto planar and nonplanar surfaces in a massively parallel manner, they are not suitable to realize microelectronic systems that consists of more than one repeating unit. Such heterogeneous systems are difficult to 
achieve due to the insufficient power of recognition of the existing self-assembly procedures. For example, in shape-directed fluid self-assembly [3], small device components settle into the holes designed to match the shape of larger components; similarly, in surface tension driven self-assembly [4]-[6], [9], the binding sites designed for one component will almost always find a partial overlap with a receptor for a different one. As a result the assembly of electrically functional heterogeneous systems that are built using nonidentical components has not been possible. There have been a few studies to overcome this problem. One successful method used geometrical shape recognition to distinguish and prealign the components, while the attachment, contact pad registration, and alignment are completed on the basis of surface tension directed self-assembly involving liquid solder [10]. The process has been applied to the parallel assemble and packaging of semiconductor dies without the use of wire bonders and robotic pick and place machines [11], [12]. Other concepts have focused on the sequential activation of selected receptors to batch transfer components on desired locations on a surface [13]-[15]. Characteristic for both methods is that batches of components are added sequentially-as opposed to adding all components at the same time. The 3-D-type heterogeneous systems are built in solution [10]-[12] while the 2.5-D-type assemblies are built through integration of the individual components on a common substrate surfaces [13]-[15].

In this paper, we describe theoretical and experimental results of a programmable and reconfigurable self-assembly (PRS) method that was first proposed conceptually one year ago [14], [15]. We adapted the liquid-solder-coated areas as receptors for self-assembly since it provides three requirements: i) they act as receptors for subunits during the assembly (no manipulator is needed), ii) they form rigid bonds upon solidification (no adhesive is needed), and iii) they provide electrical contacts to the bottom electrode of assembled devices (no wirebonder for a bottom electrode is needed). These solder-based receptors were designed to be switched "ON; liquid solder" and "OFF; solid solder" electrically by integrated heaters. This programmability of the solder-based receptors is used to batch assemble the dies. Different from prior methods [7], [8], the process does not use solder on the components themselves, and the process is designed to program the assembly of standard die geometries; the process used by Alien technology requires tapered die forms that are not commonly used by the industry. The metal contact on the component acts as binding site and attaches to activated receptors on the substrate during the fluidic self-assembly process to form an electrical and mechanical bond. We present a feasibility study by estimating the required heating power to activate a receptor and the crosstalk between receptors. Our theoretical calculations are confirmed experimentally by demonstrating the heterogeneous assembly of different semiconductor chips on flexible and curved substrates. The assembly of three nonidentical semiconductor chips (red, green, and yellow LEDs) has been accomplished. The programmable sequential assembly of multiple types of components onto target positions has been demonstrated including $300 \mu \mathrm{m}$ sized light-emitting diodes and silicon dies and the realization of a prototype color LED display segment that contains a total of 36 red, green, and yellow LED segments and 72 interconnects has been used to test the concept.

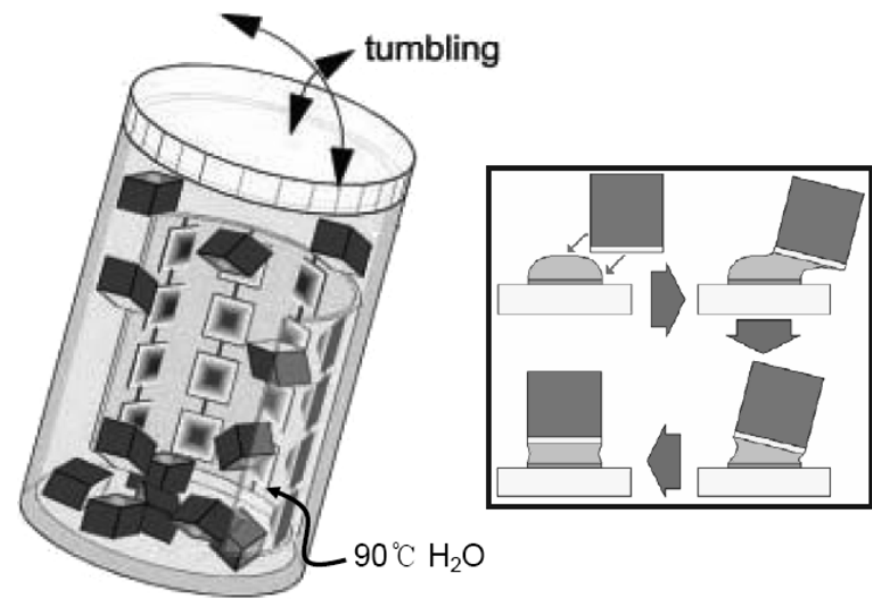

Fig. 1. Surface tension directed self-assembly. The solder coated areas on the substrate wet and bind to the metal contacts on the device segments during the self-assembly process.

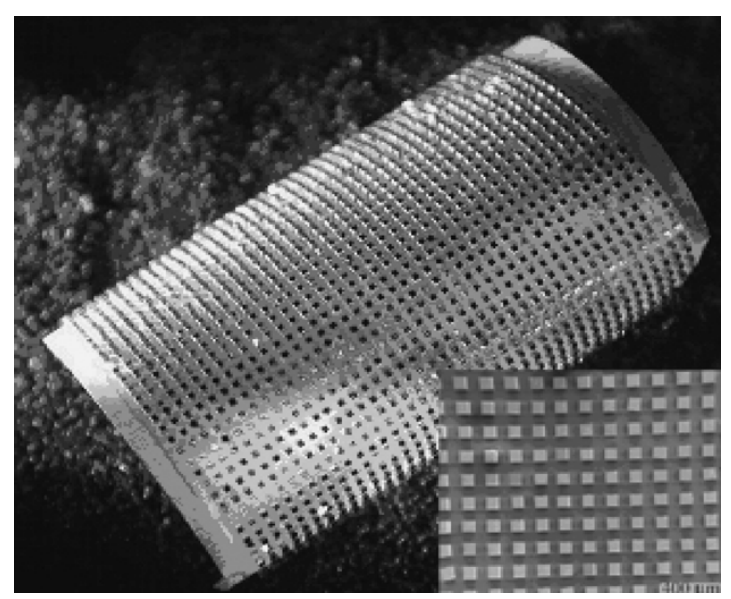

Fig. 2. Batch transfer of 1500 silicon cubes onto a curved surface.

\section{Methods}

The basic principle of the liquid-solder-receptor-directed assembly process is illustrated in Fig. 1. One side of the component is coated with a metal contact. This metal contact wets and binds to liquid-solder-based receptors during the self-assembly process at a temperature where the solder is molten. The minimization of the free surface area of the liquid solder drives the assembly into a stable, aligned position. Agitation during the assembly process occurs in the form of a periodic rotational motion. This enables the components to move around inside the glass vial, increasing the chance for components to be in contact with the molten solder. The assembly of large number of identical components has been accomplished using this process [9]. Fig. 2 illustrates results where we have assembled $\sim 1500$ silicon segments $300 \mu \mathrm{m}$ in size onto a flexible surface in $3 \mathrm{~min}$.

The basic concept of a parallel high-throughput heterogeneous self-assembly line is illustrated in Fig. 3. This concept uses a well-defined sequence instead of trying to assemble all components in a single step. The illustration shows a $3 \times 3 \mathrm{mi}-$ crosystem array (a), each of which contains four different components (b). The system is assembled by sequentially activating selected receptor sites (c). 


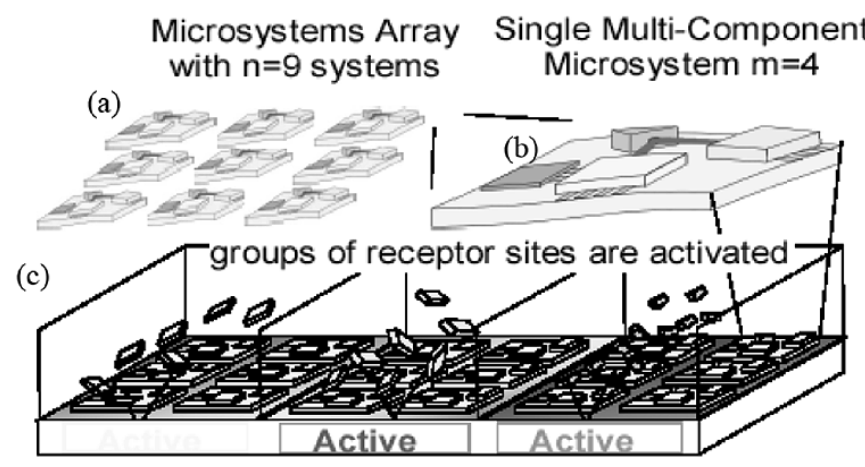

Fig. 3. Concept of the sequential self-assembly-based manufacturing process to assemble multicomponent microsystems.

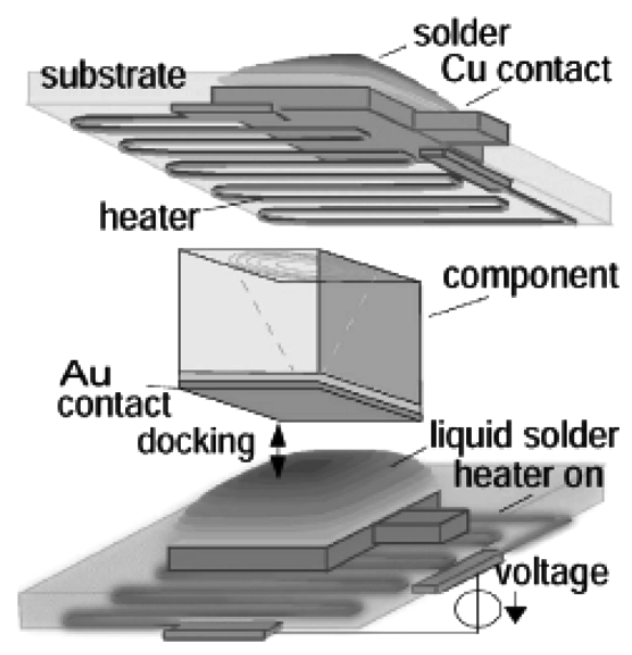

Fig. 4. The solder sites are programmed by applying an external voltage to embedded heaters.

The selective activation of the receptors enables the programmable batch transfer of desired components onto desired locations. The concept to activate the selected receptor sites is illustrated in Fig. 4. It makes use of heaters that are integrated on the back side of a flexible substrate that supports the solder-coated areas. The self-assembly of the components occurs due to the reduction of the interfacial free energy. The gold-coated binding sites of components suspended in a solution attach to solder coated areas that are heated above the melting point of the solder.

\section{SimUlATION}

To evaluate the feasibility of the proposed PRS concept, we performed thermal steady-state and transient analysis using ANSYS to estimate the necessary power to heat the selected receptors to above the melting point of the solder and the minimal spacing between receptors to prevent thermal crosstalk. We used the same dimensions and materials that were used in the experiment. The simulation is different from an earlier study that looked at the temperature distribution around line type heaters [15]. In this paper, we investigate point type heaters that are used in our experimental implementation. The point type heaters array is formed with narrow copper regions $\left(300 \times 300 \mu \mathrm{m}^{2}\right)$ that are connected with $700 \mu \mathrm{m}$ wide copper lines. The copper lines are used to connect subgroups of the

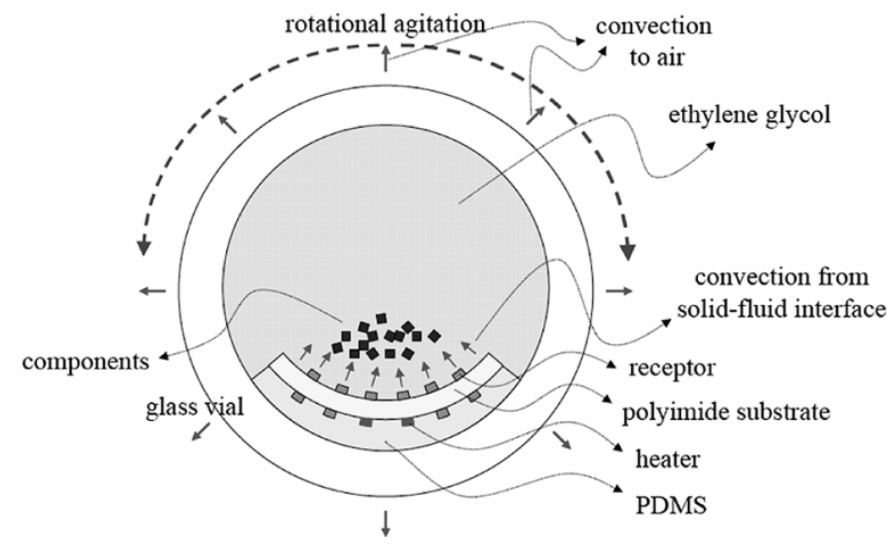

Fig. 5. Modeling of a cylindrical self-assembly.

heaters in series. The modeled substrate is a $13 \mu \mathrm{m}$ thick-polyimide film with $300 \times 300 \mu \mathrm{m}^{2}$ wide receptors on the front and $300 \times 300 \mu \mathrm{m}^{2}$ point type heaters on the back that are formed using 18.6- $\mu \mathrm{m}$-thick copper metallization. The actual self-assembly experiments were performed in a cylindrical glass vial (the inner radius $\sim 1.1 \mathrm{~cm}$; height $\sim 5 \mathrm{~cm}$ ) that was filled with ethylene glycol. The agitation during the assembly process was a periodic rotational motion between the liquid core and substrate with a peak velocity difference at the interface of $3.5 \mathrm{~m} / \mathrm{s}$ (Fig. 5). We modeled the same two-dimensional cross-sectional geometry but simplified the rotational agitation as a one-directional triangular fluid velocity distribution. We implemented a heat sink by considering a thermal convection from the glass outer boundary to air. We included thermal conduction and convection from the heater to the ethylene glycol through the polyimide and receptors [16]. We assumed the introduced heat is conducted isothermally in materials with their conduction coefficient $k_{\text {copper }}=393, k_{\text {polyimide }}=0.1549$, $k_{\text {ethyleneglycol }}=0.251 \mathrm{~W} / \mathrm{mK}$. For thermal convection, we computed the heat transfer coefficient $(h)$ at the interfaces between the substrate and ethylene glycol using a numerical calculation method inside ANSYS ( $h_{\text {copper-ethyleneglycol }}=42$, $h_{\text {polyimide-ethyleneglycol }}=27 \mathrm{~W} / \mathrm{m}^{2} \mathrm{~K} ;$ Fig. 5). The distance between the receptor on the "ON" heater and the nearest one was set to 300 and $600 \mu \mathrm{m}$, respectively.

Using this geometry and parameters, we calculated the required heating power to melt the solder and the spacing to prevent thermal crosstalk. Fig. 6 shows the calculated temperature at the heated (solid line) and unheated receptors (dashed and dotted lines) as a function of heating power. The horizontal lines indicate the melting points of the solders that we have used in our experiments. The results suggest that an applied power of $2.5 \mathrm{~mW}$ is sufficient to activate the low-melting-point solder while maintaining a temperature drop of $4.1^{\circ} \mathrm{C}(8.7 \%)$ to the nearest neighbor which is sufficient for the solder to be solid. Considering the higher melting point solder $\left(138^{\circ} \mathrm{C}\right)$, we obtained a temperature drop of $14{ }^{\circ} \mathrm{C}(10.2 \%)$.

In a second set of simulations, we studied the transient response to evaluate if heating cycles could be used. The rationale for this investigation is as follows: if the heating power exceeds the permissible range, the correct receptors would be activated first and incorrect receptors would follow later. As a 


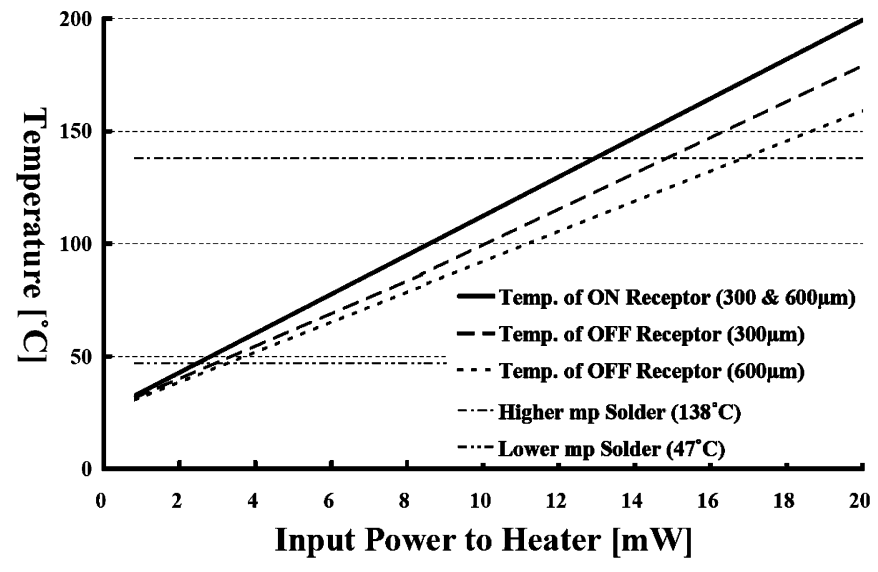

Fig. 6. Steady-state temperature as a function of heating power on the heated (-), and unheated receptors that are separated by $300(--)$ and $600 \mu \mathrm{m}(\ldots)$ wide gaps. The horizontal lines show the melting points of the solders that were used.

result there exists a time period that could be explored to assemble components without having tight controls on the heating power. In order to calculate this time period, we have performed a transient analysis using a fixed power of $6.9 \mathrm{~mW}$ that will melt neighboring receptors. Fig. 7 shows the transient response. The calculated time window is 70 and $140 \mathrm{~s}$ for gap distances of 300 and $600 \mu \mathrm{m}$, respectively, for the low-melting-point solder. A similar response was observed for higher melting point solder; it takes 470 and $690 \mathrm{~s}$ for gap distances of 300 and $600 \mu \mathrm{m}$, respectively, and a power level of $19 \mathrm{~mW}$ before the nearest neighbor becomes activated. The result suggests that heating time cycles might provide an effective method to prevent crosstalk between neighboring receptors without controlling the power level carefully.

\section{EXPERIMENTS AND DISCUSSIONS}

\section{A. Fabrication}

Under consideration of the knowledge acquired through our simulations, we proceeded to design a set of experiments for this PRS process. We designed the sequential self-assembly of three different colored LEDs on a flexible substrate for a functional display device. As a substrate material we used a polyimide film that has a copper coating on both sides (Pyralux LF7022R, Dupont, Wilmington, DE). The polyimide film is appropriate to our application since it is robust, flexible, thermally stable up to $350^{\circ} \mathrm{C}$, and compatible with standard microfabrication processes. The front side of the film was patterned for receptors and the back side with heater arrays. Using the front side copper layer, we designed the receptors with a $600 \mu \mathrm{m}$ gap distance and an electrical connection. To protect the electrical connection from being coated with solder, we masked them with a thin layer of polyimide. The back side copper was used for heaters and their electrical connections, which consisted of a copper line with two alternating widths; a high resistance $300 \times 300 \mu \mathrm{m}^{2}$ square underneath the receptors that is connected to lower resistance $700 \mu \mathrm{m}$ wide line to minimize heat generation in surrounding areas.

During the fabrication process (Fig. 8), we supported the flexible copper coated polyimide film on a polydimethylsiloxane

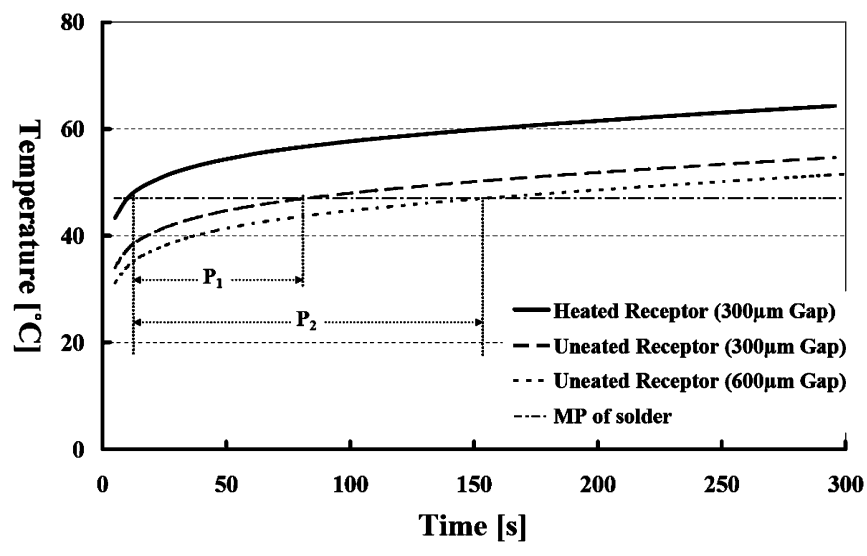

Fig. 7. Transient temperature response at the heated (-) and unheated receptor that is separated by $300(---)$ and $600 \mu \mathrm{m}(\ldots)$ gaps. The horizontal line represents the low-melting-point solder. $P_{1}(70 \mathrm{~s})$ and $P_{2}(140 \mathrm{~s})$ represent the duration where self-assembly occurs without crosstalk. If the self-assembly is not complete, a new cycle would be required with a cooled down system.

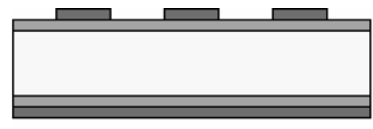

(a)

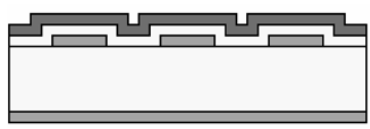

(c)

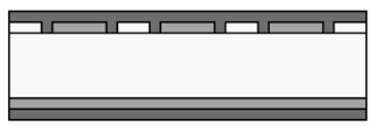

(e)

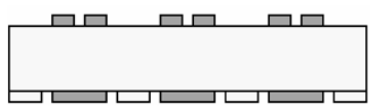

(g)

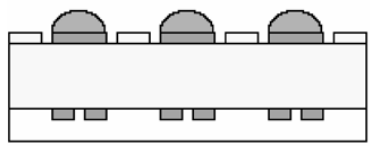

(i)

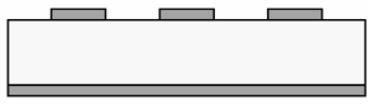

(b)

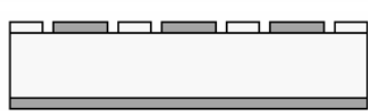

(d)

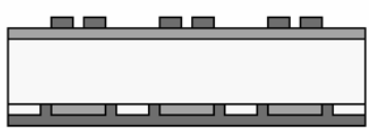

(f)

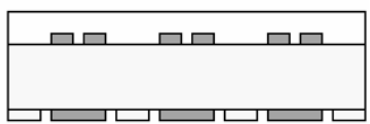

(h)

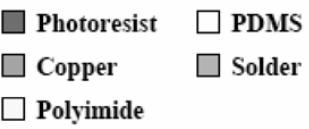

Polyimide

Fig. 8. Process flow. (a) Patterning top side for receptors; (b) copper etching; (c); polyimide and photoresist coating; (d) patterning and polyimide etching; (e) protection with thick photoresist; (f) patterning backside for heaters; (g) copper etching; (h) PDMS coating; and (i) solder coating.

(PDMS) coated silicon wafer. First, Shipley 1818 photoresist (Microposit, Phoenix, AZ) was spin-coated onto the backside of the polyimide film at $3000 \mathrm{rpm}$ for $30 \mathrm{~s}$ and baked at $105^{\circ} \mathrm{C}$ for $2 \mathrm{~min}$ to protect the copper coating during the processing of the front. Shipley 1813 photoresist (Microposit, Phoenix) was then spin-coated at $3000 \mathrm{rpm}$. After a soft bake at $105^{\circ} \mathrm{C}$ for 1 min, the substrate was exposed to ultraviolet (UV) light through a dark field mask and developed in developer 1 MIF-351: $5 \mathrm{H}_{2} \mathrm{O}$ for $12 \mathrm{~s}$. The exposed copper area was etched by an aqueous ferric chloride solution ( $1.4 \mathrm{~g}$ of $\mathrm{FeCl}_{3}$ per milliliter of $\mathrm{H}_{2} \mathrm{O}, \mathrm{pH}$ 1.3). On the patterned copper layer, we spin-coated polyimide PI2525 (HD MicroSystems, Wilmington, DE) at 3000 rpm for 
$40 \mathrm{~s}$ and baked it at $95^{\circ} \mathrm{C}$ for $50 \mathrm{~s}$ and at $130^{\circ} \mathrm{C}$ for $50 \mathrm{~s}$. Shipley 1075 was then spin-coated on top of the polyimide layer at 3000 rpm for $40 \mathrm{~s}$ and baked at $105^{\circ} \mathrm{C}$ for $5 \mathrm{~min}$. The multilayered substrate was then exposed to UV light through a dark field mask and developed in a $1 \mathrm{TMAH}(25 \%): 8 \mathrm{H}_{2} \mathrm{O}$ solution for $1 \mathrm{~min}$ to form an opening at the location of the receptors. This process not only develops the UV exposed areas but also etches the exposed PI2525 layer. After patterning the polyimide layer, the photoresist on both front and back of the film was removed using acetone and then the substrate was cured in a convection oven at $250{ }^{\circ} \mathrm{C}$ for $3 \mathrm{~h}$. Shipley 1818 photoresist was then spin-coated to protect the front during the processing of the back side. We peeled off and remounted the polyimide film upside down on the PDMS-coated silicon wafer. The same photolithography and etching sequence was applied to the copper coating on the backside of the polyimide film to define the heater array on the other side. In addition to this process we spin-coated a $150-\mu \mathrm{m}$-thick layer of PDMS onto the fabricated heater array at $500 \mathrm{rpm}$ for $30 \mathrm{~s}$ and cured it in a convection oven for $1 \mathrm{~h}$. Finally, the defined receptors were coated with solder by dipping the substrate into a liquid solder bath.

\section{B. Self-Assembly Process}

The self-assembly process was performed in a small glass vial filled with ethylene glycol (boiling point $197^{\circ} \mathrm{C}$ ). The ethylene glycol was made slightly acidic ( $\mathrm{pH} 2.5$ ) with sulfuric acid to remove metal oxides from the surface of the solder and copper binding sites. In our experiments we used two different solders (melting point $47{ }^{\circ} \mathrm{C}$, LMA-117; and melting point $138{ }^{\circ} \mathrm{C}$, LMA-281, Small Part, Miami Lakes, FL) that have been used successfully in previous self-assembly experiments [9], [10], [15]. During the assembly process, we agitated the vial by rotating it back and forth along its longitudinal axis. The angular motion was adjusted such that the components were transported to the upper half of the vial before they would drop down toward the lower half. We did not observe a notable difference between hand rotation and automatic rotation using a dc motor that was driven with a square wave ac voltage. For a $22-\mathrm{mm}$-diameter vial, back and forth rotation with amplitude of $180^{\circ}$ and $1 \mathrm{~Hz}$ frequency provided the best results.

Before completing the self-assembly experiments we tested the simulation results. The measured power to melt the lowmelting-point solder was $5.6 \mathrm{~mW}$ and 2.2 times larger than what we calculated $(2.5 \mathrm{~mW})$. The mismatch could be due to an adhesive that is located between the heater and the polyimide substrate as well as the polyimide and top copper cladding that hosts the solder. We did not include these layers in the simulation since material properties and heat conduction coefficients have not been disclosed by the manufacturer. While the required heating power was higher to melt the solder than what we expected, we obtained a close agreement for the transient response. Using heating power of $6.9 \mathrm{~mW}$ from the simulation in the experiment, we measure $105 \mathrm{~s}$ for the time interval before the neighboring receptor would be activated, which is in close agreement with the $140 \mathrm{~s}$ that was predicted by the simulation. Ethylene glycole has a boiling point of $197^{\circ}$, and we were able to observe the formation of small bubbles at the heated areas at
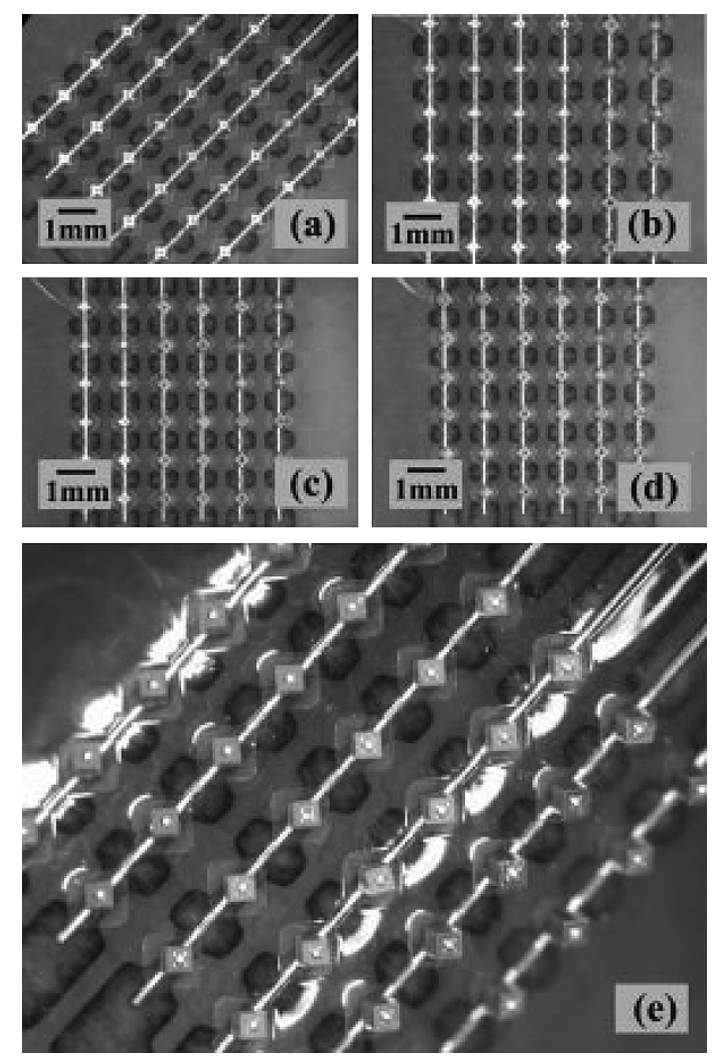

Fig. 9. Micrographs of the substrate and LEDs that have been assembled using PRS. (a) Substrate before PRS showing solder coated areas on the front and point type heaters that are formed using a $300 \mu \mathrm{m}$ narrow copper metallization that is connected to $700 \mu \mathrm{m}$ wider copper lines on the back. (b)-(d) Substrates after sequential batch assembly of (b) yellow, (c) green, and (d) red LEDs. (e) Three-dimensional view of the completed assembly after flexing the substrate.
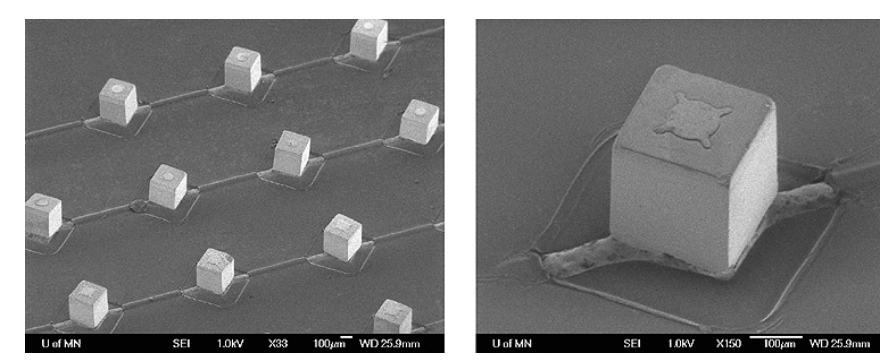

Fig. 10. The SEM pictures of the assembled substrate after three steps of PRS.

power levels exceeding $19.8 \mathrm{~mW}$, which is in close agreement with the temperature at the heated area predicted by the simulation; attachment of the components was strongly suppressed at these levels.

Based on the gained knowledge, we tested the assembly of red, green, and yellow LEDs using the transient heating approach using $6.9 \mathrm{~mW}$ in three 90 -s intervals. The commercially available LED dies (GaAsP/GaP, $280 \mu \mathrm{m}$, Knowledge*On Inc., Korea) had a four-fold symmetry and a square-shaped binding side resulting in four stable angular orientations $0,90,180$, and $270^{\circ}$. The results are summarized in Fig. 9 showing the substrate before (a) and after sequential transfer of the red (b), green (c), and yellow (d) LED dies. Fig. 10 shows an SEM picture of the self-assembled LEDs. The picture reveals the details of the polyimide masking layer and the alignment accuracy. 


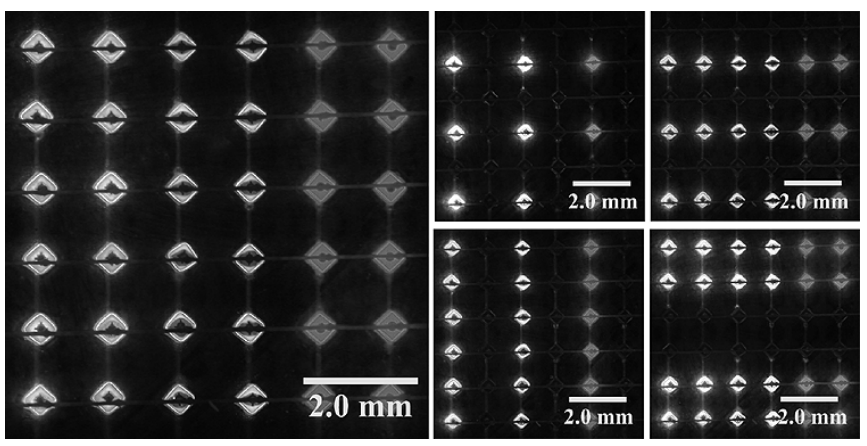

Fig. 11. The operation of the flexible display device using the PRS process with three different color LEDs.

After completion of the self-assembly, we applied a top electrode to form a functional display device. The top electrode consisted of a single-side copper-coated polyimide film (Pyralux LF7012R, Dupont, Wilmington, DE) that was structured using the same lithographic process that was used to create the receptors and heaters. We defined $60-\mu \mathrm{m}$-wide copper lines that were spaced at the same distance than the assembled diodes. All the surfaces (i.e., the gold contacts exposed on top of the LEDs and the copper wires on the top electrode) were coated with solder by dipping the assembled substrate and the top electrode into the liquid solder bath. The dip coating process did not remove the self-assembled LEDs. The assembled LEDs would stay on their receptors during the dipping process since the surface area of the top contact (the area is roughly proportional to the force of adhesion) is ten times smaller than the surface area of the bottom contact.

In order to fuse the top contacts, we positioned the flexible top electrode by hand to make contact with the anode of the LEDs. When the entire structure was heated above the melting point of the solder, the solder-coated top electrode became fused with the solder-coated anodes of the LEDs. The entire system aligned itself by adjusting the position of the components to minimize the overall interfacial energy. This process formed an electrical contact with each LED. To test the functionality of the LED display device, we applied a potential of $2 \mathrm{~V}$ between the top and substrate electrodes, which is shown in Fig. 11.

We have tested other assemblies as well. Fig. 12(a) shows the assembly results of an $M$-shaped pattern that includes two different CMOS device surrogates-300 $\mu \mathrm{m} \times 300 \mu \mathrm{m}$ and $500 \mu \mathrm{m} \times 500 \mu \mathrm{m}$ wide silicon blocks- that have been assembled on a curved surface. The $300 \mu \mathrm{m}$ sized silicon device surrogates self-assembled onto $300 \mu \mathrm{m}$ sized receptors with the heater $H 1$ being switched "ON," whereas the $500 \mu \mathrm{m}$ sized components self-assembled onto $500 \mu \mathrm{m}$ sized receptors with the heaters $H 2$ being switched "ON." Fig. 12(b) shows $280 \mu \mathrm{m}$ sized GaP-based LEDs and silicon chips that have been assembled as programmed by the underlying heaters. Fig. 12(c) shows 360 LEDs (120 red, 120 yellow, and 120 green LEDs) that have been transferred onto the desired areas as programmed using the integrated heaters without defects.

\section{DISCUSSION}

The numerical and experimental investigation have contributed to the understanding of the assembly process.

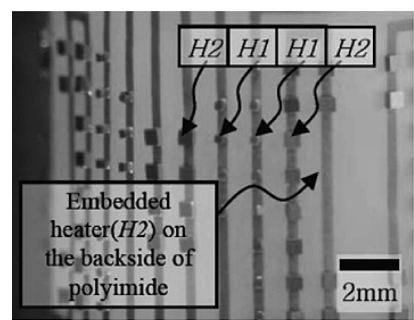

(a)

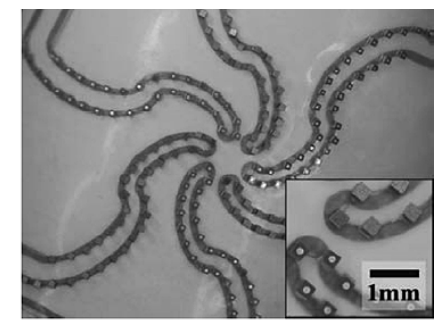

(b)

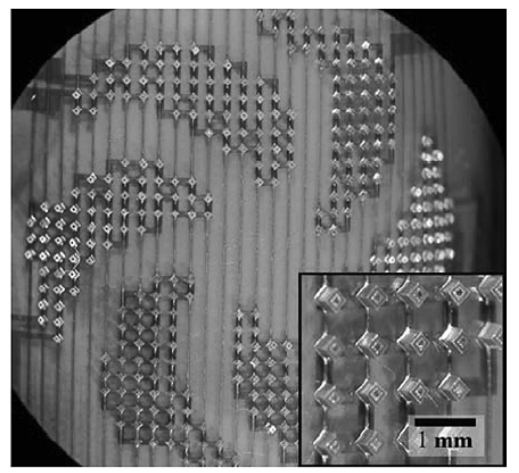

(c)

Fig. 12. Heterogeneous self-assembly programmed using linear heaters. The visible linear heater circuit is located on the back side of the flexible and translucent polyimide printed circuit board material that is wrapped around a glass cylinder. (a) shows 500- and 300- $\mu \mathrm{m}$-wide silicon device surrogates that have been transferred onto heated surface areas, whereas (b) shows $280-\mu \mathrm{m}$-wide GaAs LEDs and $300 \mu \mathrm{m}$ silicon blocks that were batch assembled onto the corresponding surface areas in a two-step self-assembly sequence - a total of 196 device segments are visible. (c) shows three different LED types - a total of 360 LEDs - that have been assembled in three successive steps without defects. The insets (b and c) show a 50- $\mu \mathrm{m}$-wide metal wire on the front of the polyimide film that connects the different device components to contact pads.

Throughout the study reported in this paper, we found the following guidelines to be effective in producing assemblies without of minimal defects.

1) The gap distance between receptors needs to be greater than the size of the components in order to avoid assembly onto adjacent receptors.

2) The gaps between receptors needs to be sufficient to prohibit heat-induced crosstalk (at least $600 \mu \mathrm{m}$ for a $300 \mu \mathrm{m}$ sized component).

3) For $<300 \mu \mathrm{m}$ sized components the connecting wires should not be coated with solder in the vicinity $(<$ $200 \mu \mathrm{m})$ of an activated receptor to prevent attachment of components.

4) The temperature on the activated receptors needs to be below the boiling point $\left(197^{\circ} \mathrm{C}\right)$ of the ethylene glycol solution to avoid the nucleation of boiling that prevents the attachment of components.

We found three types of defects and we were able to eliminate them by changing the designs: i) empty receptors, ii) receptors that captured two devices, and iii) receptors that captured the wrong device due to thermal crosstalk. The first defect [empty receptors, Fig. 13(a)] was caused by imperfections of the solder mainly due to oxidation and dissolution. We were able to remove oxidation and dissolution related defect by working at a $\mathrm{pH} 2.5$ by adding small amounts of sulfuric acid to maintain an oxide 

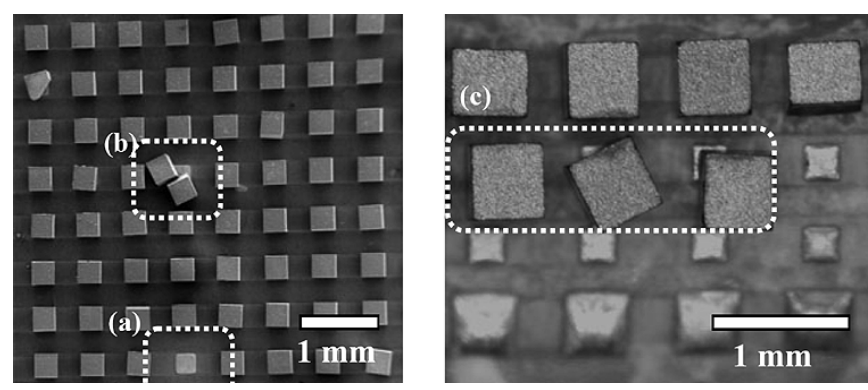

Fig. 13. Possible defects in liquid solder directed self-assembly: (a) an empty receptor, (b) a receptor occupied by two parts, and (c) assembled parts on the unheated receptors. When the first row of receptors was heated by an embedding line heater, the receptors on the second row were activated and three parts were assembled on them. SEM pictures of the assembled substrate after three steps of PSA.

free surface without dissolving the solder during the assembly process. The second defect [receptors that captured two devices, Fig. 13(b)] was caused by a large amount of solder that occupied a surface area that was twice the receptor size. It was difficult to fabricate solder-coated areas with a well-controlled volume over the entire surface area using the dip coating process and we have not yet been able to remove this defect without selecting substrates that have a uniform coating to begin with. This selection process would be inefficient in an industrial process. The last defect [receptors that captured the wrong device, Fig. 13(c)] was a defect that was related to thermal cross talk. The defect was a result of a steady temperature increase in the assembly solution due to a lack of a sufficient heat sink that finally causes an activation of neighboring receptors. We succeeded to remove this defect by i) using the time window information developed through the simulations and by ii) immersing the vial in a cool water bath to provide an adequate heat sink after each assembly step.

It was generally found to be important to use components and materials that are free of manufacturing defects to minimize defects during the self-assembly. Imperfect components will cause errors (mainly misaligned or missing components) that are not inherent to the assembly process itself. Examples include receptors that were round rather than square, receptors that were only partially coated with solder, receptors that were coated with fragments of components (these fragments can originate in the dicing process and should be filtered out using a mesh filter), receptors where the solder had dissolved, and components with partially detached metal contacts. The manufacturing-related defects can be identified quickly and prevented by ensuring that the components are good to begin with. We also observed situations where the self-assembly process was slower than expected. Consistent results were established by washing the components using acetone to remove organic contaminants and 1:100 diluted HF solution to remove surface oxides prior use.

The PRS process has been carried out for components that carry a single top and bottom contact that have a four-fold symmetry and 0, 90, 180, 270 angular orientations. We are currently investigating an approach that makes use of specific docking sites to integrate components with single angular orientation on a surface. Fig. 14 shows a first result where alignment pedestals

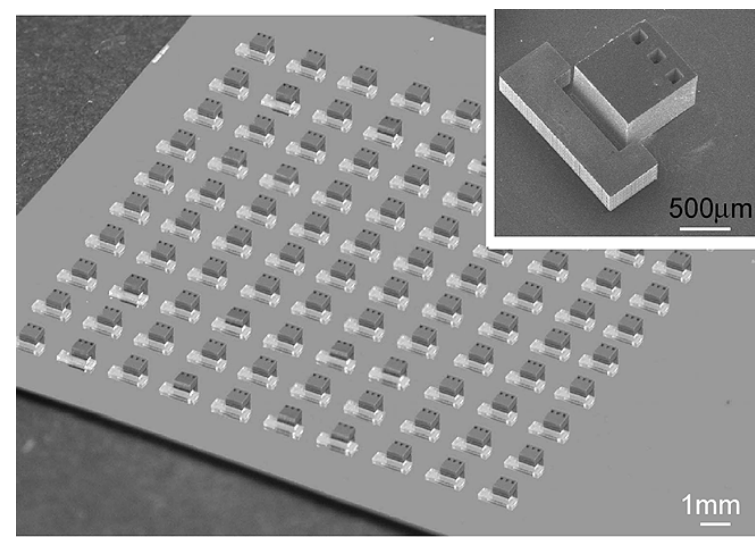

Fig. 14. Photograph and closeup SEM micrographs of 100 silicon dies that have been assembled with single angular orientation by combining SU-8 alignment pedestals with surface tension directed self-assembly involving liquid solder.

in combination with the location of the solder/binding sites have been used to accomplish angular orientation and contact pad registration. We believe that it might be possible to extend the presented PRS process to components with multiple contacts on each face if concepts of geometrical shape recognition as illustrated are included in the assembly procedure.

\section{CONCLUSION}

We have proposed and tested a programmable liquid solder driven self-assembly that could enable massive heterogeneous integration of components on planar or nonplanar surfaces. Programmability was implemented by embedding heater arrays underneath the solder receptors that can be switched "ON" and "OFF." While the feasibility has been confirmed, the technology remains in its infancy. For it to become useful, the ultimate resolution limit, component density, and throughput need to be explored. A potential disadvantage of the current implementation is that the heater structures are integrated on the substrate material. The heater structures remain there largely as overhead after the assembly. A possible solution to circumvent this overhead could be an approach that attaches the printed circuit board material onto a programmable heater plate. Nonetheless, we believe that the outlined method has a number of advantages over existing self-assembly concepts. Once fully developed, it could be used to assemble many heterogeneous systems that require electrical interconnects. The self-assembly also becomes reconfigurable, i.e., the user can make changes by changing the location of activated receptors without fabricating a new receptor array. For example, a matrix array could be used to program any desired configuration. The concept could also be combined with other sequential self-assembly methods that form three-dimensionally shaped microsystems [10].

\section{REFERENCES}

[1] J. H. Fendler, "Chemical self-assembly for electronic applications," Chem. Mater, vol. 13, pp. 3196-3210, 2001.

[2] G. M. Whitesides and B. Grzybowski, "Self-assembly at all scales," Science, vol. 295, pp. 2418-2421, 2002.

[3] J. S. Smith and H. J. J. Yeh, "Method and apparatus for fabricating selfassembling microstructures," U.S. Patent 5824 186, 1998. 
[4] K. F. Boringer, U. Srinivasan, and R. T. Howe, IEEE Conf. Micro Electro Mechanical Systems (MEMS), Interlaken, Switzerland, 2001.

[5] U. Srinivasan, M. A. Helmbrecht, C. Rembe, R. S. Muller, and R. T. Howe, "Fluidic self-assembly of micromirrors onto microactuators using capillary forces," IEEE J. Sel. Topics Quantum Electron., vol. 8, pp. 4-11, 2002.

[6] U. Srinivasan, D. Liepmann, and R. T. Howe, "Microstructure to substrate self-assembly using capillary forces," J. Microelectromech. Syst., vol. 10, pp. 17-24, 2001

[7] D. H. Gracias, J. Tien, T. L. Breen, C. Hsu, and E. M. Whitesides, "Forming electrical networks in three dimensions by self-assembly," Science, vol. 289, pp. 1170-1172, 2000.

[8] T. L. Breen, J. Tien, S. R. J. Oliver, T. Hadzic, and G. M. Whitesides, "Design and self-assembly of open, regular, 3-D mesostructures," Science, vol. 284, pp. 948-951, 1999.

[9] H. O. Jacobs, A. R. Tao, A. Schwartz, D. H. Gracias, and G. M. Whitesides, "Fabrication of a cylindrical display by patterned assembly," Science, vol. 296, pp. 323-325, 2002.

[10] W. Zheng, P. Buhlmann, and H. O. Jacobs, "Sequential shape-andsolder-directed self-assembly of functional microsystems," Proc. Nat. Acad. Sci. USA, vol. 101, pp. 12 814-12 817, 2004.

[11] W. Zheng and H. O. Jacobs, "Shape-and-solder-directed self-assembly to package semiconductor device segments," Appl. Phys. Lett., vol. 85, pp. 3635-3637, 2004.

[12] - "Fabrication of multicomponent microsystems by directed threedimensional self-assembly," Adv. Funct. Mater, vol. 15, pp. 732-738, 2005.

[13] X. Xiong, Y. Hanein, J. Fang, Y. Wang, W. Wang, D. T. Schwartz, and K. F. Bohringer, "Controlled multibatch self-assembly of microdevices," $J$. Microelectromech. Syst., vol. 12, pp. 117-127, 2003.

[14] C. R. Barry, C. J. Hoon, and H. O. Jacob, "Approaching programmable self-assembly form nanoparticle-based devices to integrated circuits," presented at the Foundations of NanoScience: Self-Assembled Architectures and Devices, Snowbird, UT, Apr. 21-23, 2004.

[15] J. Chung, W. Zheng, and H. O. Jacobs, "Programmable reconfigurable self-assembly: Approaching the parallel heterogeneous integration on flexible substrates," in IEEE Int. Conf. Micro Electro Mechanical Systems (MEMS), Miami Beach, FL, 2005.

[16] F. P. Incropera and D. P. De Witt, Introduction to Heat Transfer, 4th ed. New York: Wiley, 2002.

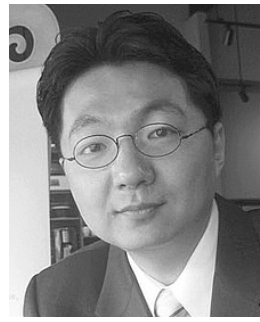

Jaehoon Chung received the B.S. and M.S. degrees in electrical engineering from Korea University, Korea, in 1996 and 1998, respectively. He is currently pursuing the $\mathrm{Ph} . \mathrm{D}$. degree in electrical engineering at the University of Minnesota-Twin Cities.

He was a MEMS Process Researcher with LG Electronics Institute of Technology. His research interests include fluidic self-assembly, microfabrication on planar and nonplanar surface, silicon optical bench, microelectronics packaging, and other topics in MEMS and NEMS device design and fabrication.

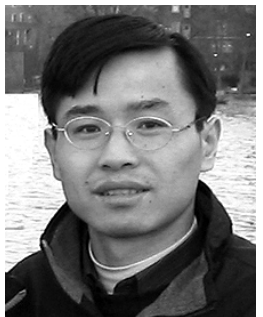

Wei Zheng (S'01-M'05) received the M.S. degree in industrial engineering from Iowa State University, Ames, in 2001. He is currently pursuing the Ph.D. degree in electrical engineering at the University of Minnesota-Twin Cities.

$\mathrm{He}$ is currently working on self-assembly-based manufacturing of heterogeneous microsystems.

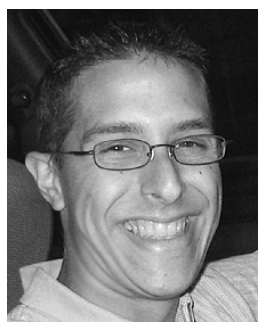

Thomas J. Hatch received the B.S. degree in mechanical engineering from the University of Puerto Rico at Mayagüez in 2003. He is currently pursuing the $\mathrm{Ph} . \mathrm{D}$. degree in mechanical engineering from the University of Minnesota-Twin Cities.

His research interests involve Computational Nanoparticle Science as well as Finite Element Analysis/Finite Element Methods as they involve nanoparticles.

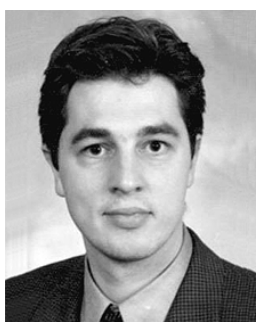

Heiko O. Jacobs (M'02) eceived the M.Sc. degree in electrical engineering from the University of Wuppertal, Germany, in 1995 and the Dr.Sc.Techn. degree in engineering from the Swiss Federal Institute of Technology (ETH) in 1999 .

He joined the Faculty at the University of Minnesota in 2001 as an Assistant Professor following a postdoctoral research position at Harvard University. During his academic career he carried out research in different departments-Chemistry (Harvard), Mechanical Engineering (ETH), Physics (ETH), and Electrical Engineering (ETH) - in interdisciplinary groups, all working in areas of micro- and nanotechnology. His research at the University of Minnesota focuses on Nontraditional micro- and nanotechnologies-integration of nanomaterials and devices across length scales and material boundaries. His current research projects are in the areas of self-assembly-based manufacturing, chip-scale heterogeneous integration, nanotechnology, nanoparticle-based devices, directed growth of nanowires, and nanometer-scale charge-based printing (nanoxerography). 